\title{
STUDY AND VALIDATION OF MESHES IN TURBULENT ISOTHERMAL PROBLEMS OF NATURAL CONVECTION IN FLAT PLATES
}

\author{
V. R. Silva ${ }^{a}$, \\ S. A. Verdério Júnior ${ }^{\text {, }}$ \\ C. Caminaga ${ }^{\mathrm{c}}$ \\ and R. C. M. Neves ${ }^{d}$ \\ a,b,c,Instituto Federal de Educação, Ciência e \\ Tecnologia de São Paulo (IFSP) \\ Departamento da Indústria \\ CEP 14804-296, Araraquara, SP, Brazil \\ avinicius@aluno.ifsp.edu.br \\ bsilvioverderio@ifsp.edu.br \\ ccaminaga@ifsp.edu.br \\ Universidade de São Paulo (USP) \\ IEE - Instituto de Energia e Ambiente \\ CEP 05508-010, São Paulo, SP, Brazil \\ driccecilio@usp.br \\ Received: Jun 16, 2021 \\ Revised: Jun 18, 2021 \\ Accepted: Jun 21, 2021
}

\section{ABSTRACT}

The study of natural convection on flat plates is of great interest in the areas of Engineering, both for the simplicity of geometry and the wide variety of applications. In the study and definition of a numerical model, an ideal mesh configuration is the one that best represents physically, with minimal numerical influence and with the lowest computational cost, the problem addressed. The influence of two mesh configurations (non-uniform staggered and entirely uniform), at different refinement levels, was studied to evaluate natural convection heat transfer rates in flat plates of $A R=5$; in isothermal conditions, in turbulent regime with the $\kappa-\omega$ SST RANS model and using free and open-source software OpenFOAM ${ }^{\circledR}$. The physical-numerical methodology applied, and the numerical results obtained were validated from experimental results in the literature. The non-uniform staggered mesh configuration proved to be more adequate in precision, and computational cost to the problem situation studied. The entirely uniform mesh proved to be infeasible due to the high number of elements and computational cost demanded.

Keywords: mesh study; natural convection; numerical analysis; numerical validation; OpenFOAM ${ }^{\circledR}$

\section{NOMENCLATURE}

AR aspect ratio

$\mathrm{CD}_{\kappa \omega} \quad \kappa-\omega S S T$ parameter

$\hat{\mathrm{C}}_{\mathrm{p}} \quad$ heat capacity at constant pressure, $\mathrm{J} /(\mathrm{kg} . \mathrm{K})$

$\mathrm{d}_{\mathrm{e}} \quad$ equivalent diameter, $\mathrm{m}$

$\overline{\text { Error }}$ average relative percentage error

$\mathrm{F}_{1} \quad$ first blending function of $\kappa-\omega S S T$ model

$\mathrm{F}_{2} \quad$ second blending function of $\kappa-\omega S S T$

$\mathrm{F}_{2}$ model

g gravity acceleration, $\mathrm{m} / \mathrm{s}^{2}$

$\mathrm{k}_{\mathrm{t}} \quad$ thermal conductivity, $\mathrm{W} /(\mathrm{m} . \mathrm{K})$

$\overline{\mathrm{Nu}}_{\mathrm{d}} \quad$ average Nusselt number, with equivalent diameter as characteristic length

$\mathrm{p}$ relative total pressure,

p_rgh relative dynamic pseudo-pressure, $\mathrm{m}^{2} / \mathrm{s}^{2}$

$\mathrm{p}_{0} \quad$ relative incompressible total pressure, $\mathrm{m}^{2} / \mathrm{s}^{2}$

$\overline{\mathrm{P}} \quad$ modified relative total pressure in the RANS model, $\mathrm{Pa}$

Pr Prandtl number

$\mathrm{Pr}_{\mathrm{t}} \quad$ turbulent Prandtl number

$\overline{q^{\prime \prime}} \quad$ average convective heat flux over the plate, $\mathrm{W} / \mathrm{m}^{2}$.

Rayleigh number, with equivalent diameter

$\mathrm{Ra}_{\mathrm{d}_{\mathrm{e}}} \quad$ as characteristic length

$\mathrm{S} \quad$ specific strain rate tensor module, $s^{-1}$

$\mathrm{T} \quad$ temperature field OpenFOAM ${ }^{\circledR}$ parameter,

$\mathrm{T}_{\mathrm{REF}}$ $\mathrm{K}$ average reference temperature, $\mathrm{K}$

\begin{tabular}{|c|c|}
\hline$\overline{\mathrm{T}}$ & $\begin{array}{l}\text { average component of temperature in the } \\
\text { RANS model, } \mathrm{K}\end{array}$ \\
\hline $\mathrm{T}_{\infty}$ & free-stream temperature, $\mathrm{K}$ \\
\hline$\overline{\mathrm{u}}_{\mathrm{i}}, \overline{\mathrm{u}}_{\mathrm{j}}$ & $\begin{array}{l}\text { indicial velocity average components in the } \\
\text { RANS model, } \mathrm{m} / \mathrm{s}\end{array}$ \\
\hline $\mathrm{U}$ & velocity field OpenFOAM ${ }^{\circledR}$ parameter, $\mathrm{m} / \mathrm{s}$ \\
\hline$\overline{\mathrm{x}}_{1}, \overline{\mathrm{x}}_{\mathrm{J}}$ & indicial coordinates, $\mathrm{m}$ \\
\hline y & $\begin{array}{l}\text { the distance from the field point to the } \\
\text { nearest wall, } m\end{array}$ \\
\hline $\mathrm{Z}$ & elevation, m \\
\hline
\end{tabular}

\section{Greek symbols}

$\alpha_{\mathrm{t}} \quad$ turbulent thermal diffusivity, $\mathrm{m}^{2} / \mathrm{s}$

$\alpha^{*}$ coefficient combination function of $\kappa-\omega S S T$

$\alpha^{*} \quad$ model, in $\alpha_{1}^{*}$ and $\alpha_{2}^{*}$ forms

$\beta \quad$ thermal coefficient of volume expansion, $\mathrm{K}^{-1}$

$\beta^{*} \quad \kappa-\omega$ SST empirical constant

$\beta^{\prime} \quad \kappa-\omega$ SST empirical constant

$\Delta \mathrm{T}$ temperature difference between the plate and the medium, $\mathrm{K}$

$\varepsilon \quad$ dissipation rate of turbulent kinetic energy, $\mathrm{m}^{2} / \mathrm{s}^{3}$

$\kappa \quad$ turbulent kinetic energy, $\mathrm{m}^{2} / \mathrm{s}^{2}$

$\mu \quad$ dynamic viscosity, $\mathrm{kg} /(\mathrm{m} . \mathrm{s})$

$\mu_{\mathrm{t}} \quad$ turbulence dynamic viscosity, $\mathrm{kg} /(\mathrm{m} . \mathrm{s})$

$v \quad$ kinematic viscosity, $\mathrm{m}^{2} / \mathrm{s}$

$v_{\mathrm{t}} \quad$ turbulence kinematic viscosity, $\mathrm{m}^{2} / \mathrm{s}$

$\bar{\rho} \quad$ fluid density evaluated in $\overline{\mathrm{T}}, \mathrm{kg} / \mathrm{m}^{3}$

$\sigma_{\mathrm{k}}^{*} \quad \kappa-\omega \mathrm{SST}$ empirical constant 
$\sigma_{\omega} \quad \kappa-\omega$ SST empirical constant, in $\sigma_{\omega 1}$ and $\sigma_{\omega 2}$ forms

$\omega$ specific dissipation rate of turbulent kinetic energy, $s^{-1}$

$\nabla \quad$ gradient operator

\section{INTRODUCTION}

Natural convection occurs due to the combined presence of density gradients in the fluid (due to the existence of temperature gradients) and gravitational forces (proportional to density), which generate buoyant forces, which are responsible for the flow (Bergman et al., 2014).

Natural convection has a significant influence on operating temperatures in power generation devices and electronics (Bergman et al., 2014). In the quest to improve thermal efficiency and ensure greater applicability of natural convection cooling in current technologies, studies and research are increasingly being developed in industry and the academic community, represented by the works of: Machado and Ramos (2006), in numerical simulation from natural convection inside a thermal diode, for feasibility analysis in building optimization applications; Mariani and Belo (2006), in the numerical thermal fluid study of natural convection in a $2 \mathrm{D}$ square cavity, in a laminar regime with Rayleigh numbers from $10^{4}$ to $10^{6}$; Padilla et al. (2006), in the numerical analysis of natural convection in cylindrical horizontal rings, under fixed temperature conditions on the surfaces and in low and moderate Rayleigh numbers; Kitamura et al. (2015), in the experimental study of natural convection in isothermal flat plates, with the survey of empirical correlations of $\overline{\mathrm{Nu}}_{\mathrm{d}_{\mathrm{e}}} \times \mathrm{Ra}_{\mathrm{d}_{\mathrm{e}}}$ in the laminar and turbulent regimes; Silva et al. (2016), in the study of steady-state heat transfer by natural convection and thermal radiation in heat sinks with rectangular fins (vertical and horizontal); Frank et al. (2019), in the study of cooling of electronic components, using OpenFOAM ${ }^{\circledR}$ and considering the mechanisms of heat transfer by natural convection and thermal radiation.

In the construction of numerical models, several hypotheses and parameters are described to represent the studied physical application, with the resolution of the mathematical model for each discretized element of the domain. In general, increasing the number of elements in the mesh improves the accuracy of the solutions (Tu et al., 2008). However, this takes more time and computing resources (Norton and Sun, 2006). In the search for lower computational costs, with good precision of results, it is necessary to choose the ideal mesh that provides these characteristics.

Boz et al. (2014) studied the effects of mesh refinement and time lag reduction on the accuracy of a CFD solution. They found that the refinement of the mesh significantly affected the temperature distribution, which led to lower precision of the results. Verdério Júnior et al. (2015), in the numerical investigation of the effects of turbulence modeling in a continuous furnace with indirect heating, through tests of independence and consistency of meshes and cost-benefit analyses, demonstrated the advantage of using a less refined mesh. In well as the work by Bilus et al. (2013) in correlated numerical models for the analysis of cavitation flows, the use of a less refined mesh proved to be equally satisfactory regarding the uncertainty about more refined models.

This work aims to study the influence of two different mesh configurations - non-uniform staggered and entirely uniform - on the prediction of turbulent natural convection heat transfer rates in isothermal flat plates. It also objectives to validate the modeling methodology and numerical analysis proposed through experimental results from Kitamura et al. (2015). The exclusive use of free and open-source numerical tools is another differential of this work, demonstrating alternatives for conducting CFD research under conditions without significant financial resources and/or state-of-the-art infrastructure.

\section{PROBLEM FORMULATION}

\section{Physical Model}

The geometry studied in this work is a flat isothermal plate at $313.15 \mathrm{~K}$, centered at the bottom of an open physical domain, with dimensions $4 \mathrm{x} 4 \mathrm{x}$ $4 \mathrm{~m}$ and filled with air at $\mathrm{T}_{\infty}=293.15 \mathrm{~K}$. The plate has a length of $0.4472 \mathrm{~m}$, width of $2.2361 \mathrm{~m}$, aspect ratio (AR) equal to 5 , area of $1 \mathrm{~m}^{2}$, and equivalent diameter $\left(\mathrm{d}_{\mathrm{e}}\right)$ to $0.7454 \mathrm{~m}$.

From the double symmetry characteristics of the physical domain about the $y$ and $z$ axes and to reduce the computational cost of the simulations, a computational domain of a quarter of the total domain was defined; as shown in Fig. 1.

The computational domain was divided into different regions to identify and implement the boundary conditions. The regions are:

- $\operatorname{TOP}(\mathrm{z}=4)$, BACK $(\mathrm{y}=2)$, RIGHT $(\mathrm{x}=2)$ and BOTTOM $(z=0)$ : open regions, with transport of mass and energy across boundaries.

- $\operatorname{LEFT}(\mathrm{x}=0)$ and FRONT $(\mathrm{y}=0)$ : regions of the symmetry planes.

- PLATE $(z=0)$ : solid wall region.

The free and open-source software SALOME, version 9.6.0, was used to generate the CAD model in Fig. 1. Through the generated model and using the cfMesh library, version 1.1, has the construction of the different meshes studied in this work.

Two different configurations of threedimensional Cartesian hexahedral meshes were studied at different levels of refinement. Mesh A is composed of three different staggered levels, with decreasing refinement from the plate region, which presents a localized uniform refinement with $5 \mathrm{~mm}$ 
edge elements. Mesh B, in turn, is composed of a uniform homogeneous refinement.

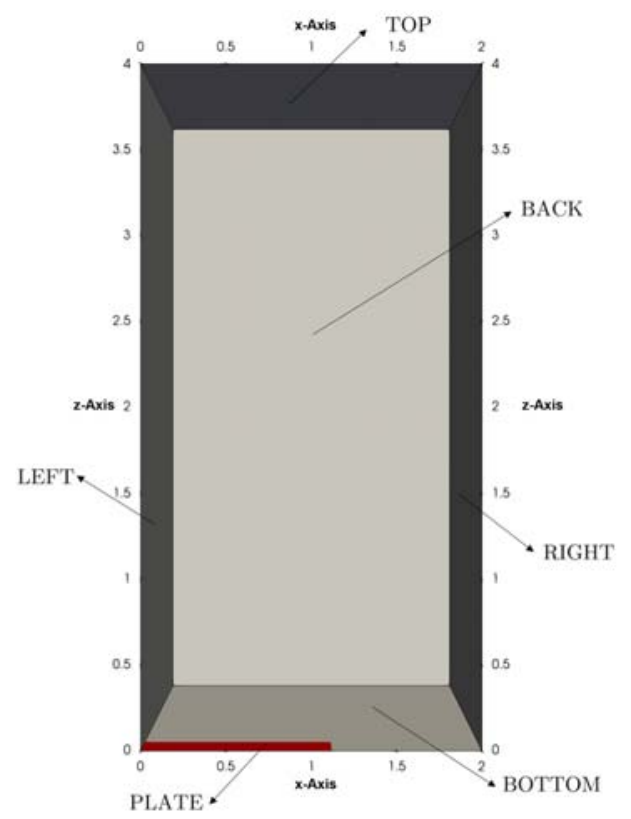

Figure 1. Schematic representation of the front view of the computational model with boundaries identification.

The different degrees of refinement used in the different mesh configurations were defined through the maxCellSize parameter, which according to Juretic (2015), characterizes the default or maximum size of mesh elements. Table 1 shows the variations of this parameter used and the respective characteristics of the meshes constructed and studied in this work.

Table 1. Characteristics of the meshes used.

\begin{tabular}{c|c|c|c}
\hline \multicolumn{2}{c|}{ MESH A } & \multicolumn{2}{c}{ MESH B } \\
\hline maxCellSize & Elements & maxCellSize & Elements \\
\hline 10.00 & 96626 & 0.250 & 1024 \\
\hline 5.00 & 108555 & 0.200 & 2000 \\
\hline 2.50 & 194708 & 0.100 & 16128 \\
\hline 2.00 & 313746 & 0.075 & 42375 \\
\hline 1.00 & 1492370 & 0.050 & 128039 \\
\hline 0.90 & 2044144 & 0.025 & 1024039 \\
\hline 0.80 & 2807540 & 0.015 & 4812247 \\
\hline
\end{tabular}

Figs. 2 and 3 illustrate Mesh A and B at their lowest and highest levels of refinement, respectively, with the highest and lowest maxCellSize. Note that for the largest parameters, the refinement of the mesh is smaller and, therefore, a smaller number of larger elements compose the mesh. The opposite happens for the smallest parameters, where there is a greater refinement and, therefore, a larger number of smaller elements compose the mesh.

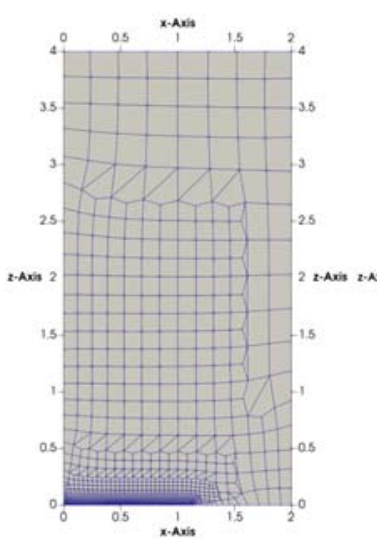

(a)

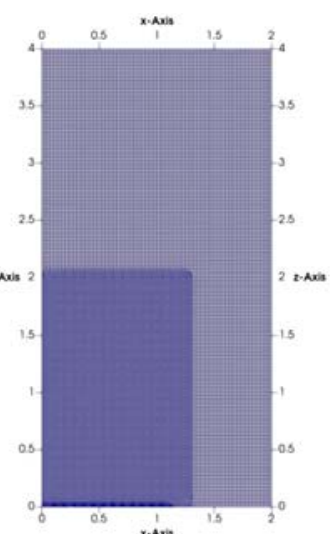

(b)

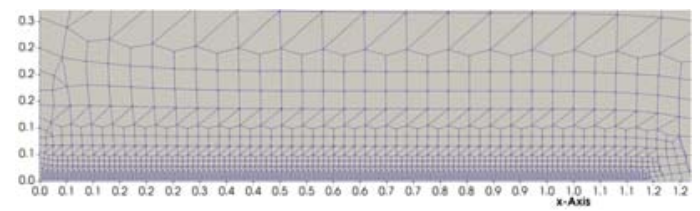

(c)

Figure 2. Visual comparison of Mesh A (a) less refined $($ maxCellSize $=10.00)$, $(b)$ more refined $(\max C e l l S i z e=0.80)$ and of the $(c)$ detail view of the plate region refinement.

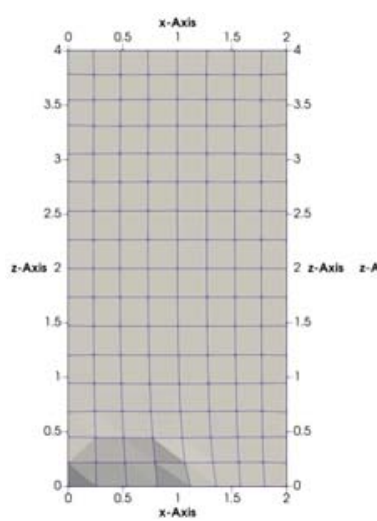

(a)

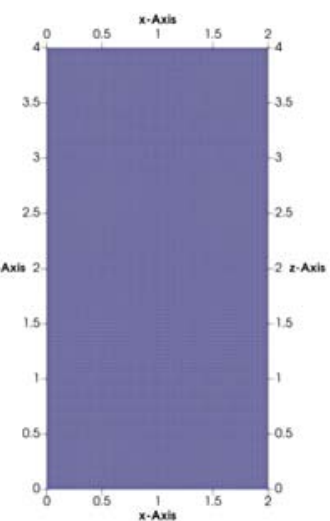

(b)
Figure 3. Visual comparison of Mesh B (a) less refined $(\operatorname{maxCellSize}=0.250)$ and $(\mathrm{b})$ more refined $($ maxCellSize $=0.015)$.

From Fig. 3, it is possible to identify the presence of a region of spatial geometric distortion in the less refined mesh, which tends to impair the numerical stability, convergence, and accuracy of the numerical solutions.

\section{Mathematical and Numerical Model}


In selecting the governing equations and defining the mathematical model to be solved, several simplifying hypotheses and physical models were adopted. Based on the physics of the problem and the typical conditions established in Pope (2000), Bird et al. (2004) and Versteeg and Malalasekera (2017):

- Physical properties constant and evaluated in $\mathrm{T}_{\mathrm{REF}}=293.15 \mathrm{~K}$, that are: $v=1.6207 \cdot 10^{-5} \mathrm{~m}^{2} / \mathrm{s}$, $\bar{\rho}=1.1509 \mathrm{~kg} / \mathrm{m}^{3}, \quad \widehat{\mathrm{C}}_{\mathrm{p}}=1007.1260 \mathrm{~J} /(\mathrm{kg} \cdot \mathrm{K})$, $\beta=0.00323 \mathrm{~K}^{-1}, \operatorname{Pr}=0.7066, \operatorname{Pr}_{\mathrm{t}}=0.85$, and $\mathrm{k}_{\mathrm{t}}=26.5331 \cdot 10^{-3} \mathrm{~W} /(\mathrm{m} \cdot \mathrm{K})$.

- Air as a Newtonian fluid, with the density changes according to the Boussinesq approximation for inclusion of the buoyant forces.

- Turbulent flow with $\mathrm{Ra}_{\mathrm{d}_{\mathrm{e}}}=3 \cdot 10^{8}$, according to the classification of Kitamura et al. (2015). Condition implemented through numerical and virtual adjustment of the vertical $\mathrm{z}$ component of gravity acceleration, with the value $\vec{g}=(0,0,-4.0824) \mathrm{m} / \mathrm{s}^{2}$. - Thermal radiation heat transfer can be disconsidered.

- Continuity Equation in turbulent regime:

$$
\frac{\partial \overline{\mathrm{u}}_{\mathrm{i}}}{\partial \mathrm{x}_{\mathrm{i}}}=0
$$

- Momentum Equation in turbulent regime, according to Boussinesq's turbulent viscosity hypothesis:

$$
\begin{aligned}
\bar{\rho} \overline{\mathrm{u}}_{\mathrm{j}} \frac{\partial \overline{\mathrm{u}}_{\mathrm{i}}}{\partial \mathrm{x}_{\mathrm{j}}} & =\frac{\partial}{\partial \mathrm{x}_{\mathrm{j}}}\left[\left(\mu+\mu_{\mathrm{t}}\right)\left(\frac{\partial \overline{\mathrm{u}}_{\mathrm{i}}}{\partial \mathrm{x}_{\mathrm{j}}}+\frac{\partial \overline{\mathrm{u}}_{\mathrm{j}}}{\partial \mathrm{x}_{\mathrm{i}}}\right)\right] \\
& -\frac{\partial \overline{\mathrm{P}}}{\partial \mathrm{x}_{\mathrm{i}}}-\bar{\rho} \mathrm{g}_{\mathrm{i}} \beta\left(\overline{\mathrm{T}}-\mathrm{T}_{\infty}\right)
\end{aligned}
$$

- Energy Equation in turbulent regime:

$$
\frac{\partial}{\partial \mathrm{x}_{\mathrm{j}}}\left(\bar{\rho} \overline{\mathrm{u}}_{\mathrm{j}} \overline{\mathrm{T}}\right)=\frac{\partial}{\partial \mathrm{x}_{\mathrm{j}}}\left[\left(\frac{\mu}{\operatorname{Pr}}+\frac{\mu_{\mathrm{t}}}{\operatorname{Pr}_{\mathrm{t}}}\right)\left(\frac{\partial \overline{\mathrm{T}}}{\partial \mathrm{x}_{\mathrm{j}}}\right)\right]
$$

- Use of SIMPLE algorithm for pressure-velocitytemperature coupling of transport equations.

- Transient simulation until solution convergence to steady state.

- Turbulence modeling using the RANS $\kappa-\omega$ SST model, according to Menter et al. (2003) and described by Table 2 and equations:

$$
\begin{aligned}
& \overline{\mathrm{u}}_{\mathrm{j}} \frac{\partial \kappa}{\partial \mathrm{x}_{\mathrm{j}}}= \min \left[v_{\mathrm{t}} \frac{\partial \overline{\mathrm{u}}_{\mathrm{i}}}{\partial \mathrm{x}_{\mathrm{j}}}\left(\frac{\partial \overline{\mathrm{u}}_{\mathrm{i}}}{\partial \mathrm{x}_{\mathrm{j}}}+\frac{\partial \overline{\mathrm{u}}_{\mathrm{j}}}{\partial \mathrm{x}_{\mathrm{i}}}\right) ; 10 \beta^{*} \kappa \omega\right] \\
&-\beta^{*} \kappa \omega+\frac{\partial}{\partial \mathrm{x}_{\mathrm{j}}}\left[\left(v+\sigma_{\kappa}^{*} v_{\mathrm{t}}\right) \frac{\partial \kappa}{\partial \mathrm{x}_{\mathrm{j}}}\right] \\
& \overline{\mathrm{u}}_{\mathrm{j}} \frac{\partial \omega}{\partial \mathrm{x}_{\mathrm{j}}}=\alpha^{*} S^{2}-\beta^{\prime} \omega^{2}+\frac{\partial}{\partial \mathrm{x}_{\mathrm{j}}}\left[\left(v+\sigma_{\omega} v_{\mathrm{t}}\right) \frac{\partial \omega}{\partial \mathrm{x}_{\mathrm{j}}}\right] \\
&+2\left(1-\mathrm{F}_{1}\right) \sigma_{\omega 1} \frac{1}{\omega} \frac{\partial \kappa}{\partial \mathrm{x}_{\mathrm{j}}} \frac{\partial \omega}{\partial \mathrm{x}_{\mathrm{j}}}
\end{aligned}
$$

$$
\begin{gathered}
\mathrm{S}=\frac{\sqrt{2}}{2}\left(\frac{\partial \overline{\mathrm{u}}_{\mathrm{i}}}{\partial \mathrm{x}_{\mathrm{j}}}+\frac{\partial \overline{\mathrm{u}}_{\mathrm{j}}}{\partial \mathrm{x}_{\mathrm{i}}}\right) \\
\mathrm{F}_{1}=\tanh \left\{\left\{\min \left[\max \left(\frac{\sqrt{\kappa}}{\beta^{*} \omega \mathrm{y}}, \frac{500 v}{\mathrm{y}^{2} \omega}\right), \frac{4 \bar{\rho} \sigma_{\omega 2} \mathrm{\kappa}}{\mathrm{CD} \mathrm{\kappa}_{\mathrm{K}} \mathrm{y}^{2}}\right]\right\}^{4}\right\} \\
\mathrm{CD}_{\kappa \omega}=\max \left(2 \bar{\rho} \sigma_{\omega 2} \frac{1}{\omega} \frac{\partial \kappa}{\partial \mathrm{x}_{\mathrm{j}}} \frac{\partial \omega}{\partial \mathrm{x}_{\mathrm{j}}}, 10^{-10}\right) \\
\mathrm{F}_{2}=\tanh \left[\left[\max \left(\frac{2 \sqrt{\kappa}}{\beta^{*} \omega \mathrm{y}}, \frac{500 v}{\mathrm{y}^{2} \omega}\right)\right]^{2}\right] \\
v_{\mathrm{t}}=\frac{0.3 \kappa}{\max \left(0.3 \omega ; \mathrm{SF}_{2}\right)} \\
\alpha_{1}^{*}=\alpha_{1}^{*} \mathrm{~F}_{1}+\alpha_{2}^{*}\left(1-\mathrm{F}_{1}\right)
\end{gathered}
$$

Table 2. Empirical conditions used in the turbulence model k- $\omega$ SST, according Menter et al. (2003).

\begin{tabular}{c|c|c|c|c}
\hline Constants & $\sigma_{\mathrm{k}}^{*}$ & $\sigma_{\omega}$ & $\beta^{\prime}$ & $\alpha^{*}$ \\
\hline$\alpha_{1}^{*}(\kappa-\varepsilon)$ & 0.85 & 0.5 & 0.075 & $5 / 9$ \\
\hline$\alpha_{2}^{*}(\kappa-\omega)$ & 1.0 & 0.856 & 0.0828 & 0.44 \\
\hline
\end{tabular}

- Use of the Finite Volume Method to solve discretized transport equations, implemented in OpenFOAM ${ }^{\circledR}$ software, version 2012.

- Modeling and numerical structuring of the

\begin{tabular}{|c|c|c|c|}
\hline Regions & $\mathrm{T}[\mathrm{K}]$ & $\mathrm{U}[\mathrm{m} / \mathrm{s}]$ & $\begin{array}{c}\text { p_rgh } \\
{\left[\mathrm{m}^{2} / \mathrm{s}^{2}\right]}\end{array}$ \\
\hline PLATE & $\begin{array}{c}\text { fixed Value } \\
\text { uniform } \\
313.15\end{array}$ & noSlip & $\begin{array}{l}\text { fixedFlux } \\
\text { Pressure }\end{array}$ \\
\hline TOP & \multirow{4}{*}{ inletOutlet } & \multirow{4}{*}{$\begin{array}{c}\text { pressure } \\
\text { InletOutlet } \\
\text { Velocity }\end{array}$} & \multirow{4}{*}{$\begin{array}{c}\text { total } \\
\text { Pressure }\end{array}$} \\
\hline BACK & & & \\
\hline RIGHT & & & \\
\hline BOTTOM & & & \\
\hline LEFT & \multicolumn{3}{|c|}{ symmetry } \\
\hline FRONT & \multicolumn{3}{|c|}{ symmetry } \\
\hline
\end{tabular}
problem using the buoyantBoussinesqSimpleFoam soler, according to Moukalled et al. (2015) and OpenCFD (2020).

\section{Boundary Conditions}

The boundary conditions were defined according to the real physical conditions of the physical model in symmetry in Fig. 1. Table 4, using typical commands from OpenFOAM $\AA$, illustrates the conditions implemented in each region. The physical equivalence of these conditions is detailed throughout this section, as per the references Moukalled et al. (2015) and OpenCFD (2020).

Table 3. Boundary conditions. 
Table 3. (cont.) Boundary conditions.

\begin{tabular}{|c|c|c|c|}
\hline Regions & $\mathrm{p}\left[\mathrm{m}^{2} / \mathrm{s}^{2}\right]$ & $\alpha_{t}\left[\mathrm{~m}^{2} / \mathrm{s}\right]$ & $\kappa\left[\mathrm{m}^{2} / \mathrm{s}^{2}\right]$ \\
\hline PLATE & & $\begin{array}{c}\text { alphat } \\
\text { Jayatilleke } \\
\text { Wall } \\
\text { Function } \\
\text { uniform } 0\end{array}$ & $\begin{array}{c}\text { kqRWall } \\
\text { Function } \\
\text { uniform } \\
1 e-05\end{array}$ \\
\hline TOP & calculated & \multirow{4}{*}{\multicolumn{2}{|c|}{ zeroGradient }} \\
\hline BACK & & & \\
\hline RIGHT & & & \\
\hline ВОTТОМ & & & \\
\hline LEFT & \multicolumn{3}{|c|}{ symmetry } \\
\hline FRONT & \multicolumn{3}{|c|}{ symmetry } \\
\hline
\end{tabular}

Table 3. (cont.) Boundary conditions.

\begin{tabular}{c|c|c}
\hline Regions & $v_{t}\left[\mathrm{~m}^{2} / \mathrm{s}\right]$ & $\omega\left[\mathrm{s}^{-1}\right]$ \\
\hline PLATE & $\begin{array}{c}\text { nutkWallFunction } \\
\text { uniform 0 }\end{array}$ & $\begin{array}{c}\text { omegaWallFunction } \\
\text { uniform 1e-08 }\end{array}$ \\
\cline { 1 - 1 } TOP & \multicolumn{2}{|}{ zeroGradient } \\
\cline { 1 - 1 } BACK & \multicolumn{2}{|c}{ symmetry } \\
\cline { 1 - 1 } BOTTOM & \multicolumn{2}{|c}{ symmetry } \\
\cline { 1 - 1 } LEFT & \multicolumn{2}{|c}{}
\end{tabular}

The calculated condition provides a calculation condition of $\mathrm{p}$ from the dynamic pseudo-pressure field $p_{-}$rgh. With the definitions of $p=\bar{P} / \bar{\rho}$ and $\mathrm{p}_{-}$rgh $=\mathrm{p}-|\mathbf{g}| \mathrm{z}$.

For p_rgh, the condition fixedFluxPressure defines $\nabla p$ _rgh to satisfy the condition set out in the U. In turn, totalPressure is a condition of total pressure, given for an incompressible flow by $\mathrm{p}=$ $\mathrm{p}_{0}-0.5|\mathrm{U}|^{2}$.

For the velocity field $\mathrm{U}$, the noSlip condition sets the velocity to zero. In turn, the pressureInletOutletVelocity condition defines $\nabla \mathrm{U}=$ 0 for output regions, and for input regions, there is the calculation of the normal components of $U$ from p_rgh.

For temperature field $\mathrm{T}$, fixedValue and inletOutlet conditions were defined. The first sets the temperature to $313.15 \mathrm{~K}$, and the second applies the condition $\nabla \mathrm{T}=0$ for output regions and $\mathrm{T}=\mathrm{T}_{\infty}$ for input regions.

The omegaWallFunction condition provides a wall constraint in $\omega$ for turbulence models with Reynolds low and high. The nutkWallFunction condition provides a wall constraint on $v_{t}$, based on $\kappa$. The kqRWallFunction condition provides a condition of $\nabla \kappa=0$ for cases of flow with high Reynolds number. The condition alphatJayatillekeWallFunction provides a thermal wall function for $\alpha_{t}$, based on the Jayatilleke model and according to Jayatilleke (1966).
Finally, the zeroGradient and symmetry conditions define null gradient and symmetry restriction, respectively.

\section{RESULTS AND DISCUSSION}

The validation and accuracy analysis of the numerical results obtained in the simulations made in this work were based on the experimental results of Kitamura et al. (2015), having the value of $\overline{\mathrm{Nu}}_{\mathrm{d}_{\mathrm{e}_{\mathrm{EXP}}}}=84,6806$ for $\mathrm{Ra}_{\mathrm{d}_{\mathrm{e}}}=3 \cdot 10^{8}$ as a reference. The evaluation of the $\overline{\mathrm{Nu}}_{\mathrm{d}_{\text {e NUMERICAL }}}$ of the simulations occurred through the equation:

$$
\overline{\mathrm{Nu}}_{\mathrm{d}_{\text {NUMERICAL }}}=\frac{\overline{\mathrm{q}^{\prime \prime}} \cdot \mathrm{d}_{\mathrm{e}}}{\Delta \mathrm{T} \cdot \mathrm{k}_{\mathrm{t}}}
$$

, with the average convective heat flux over the plate $\left(\overline{q^{\prime \prime}}\right)$ obtained through the OpenFOAM ${ }^{\circledR}$ 's utility wallHeatFluxIncompressible.

The accuracy of the numerical results obtained, compared to experimental results in the literature, occurs through the calculation of the average relative percentage error, according to the equation:

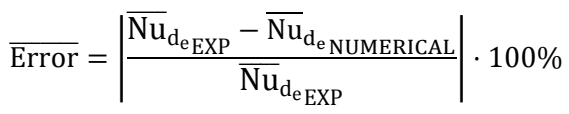

To study the different meshes constructed in configurations $\mathrm{A}$ and $\mathrm{B}$, as shown in Table 1, and analyze the accuracy of the numerical results obtained in relation to the reference by Kitamura et al. (2015), we used the free library Matplotlib, version 3.2.1 and written in python language, for graphical plotting of the obtained results.

The graphs in Fig. 4 and 5 show the evolution of $\overline{\mathrm{q}^{\prime \prime}}$ with the number of iterations performed for the different numerical models made for Meshes A and $\mathrm{B}$, respectively. The analysis of these curves shows the characteristics of numerical stability and convergence of the simulations performed.

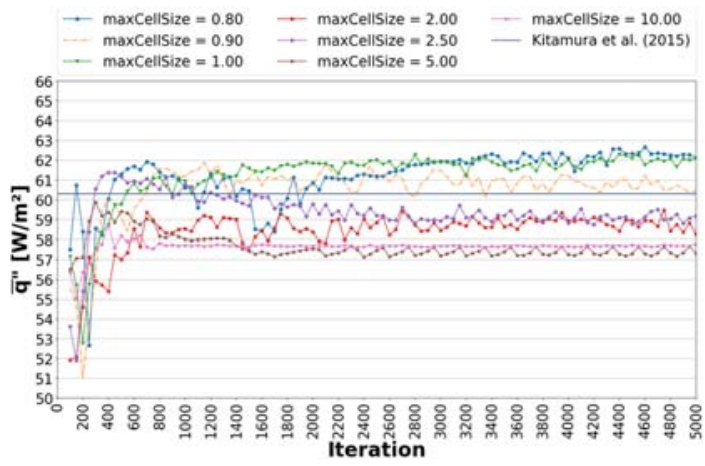

Figure 4. Average convective heat flux over the plate versus iterations number for the different refinement degrees of Mesh A. 


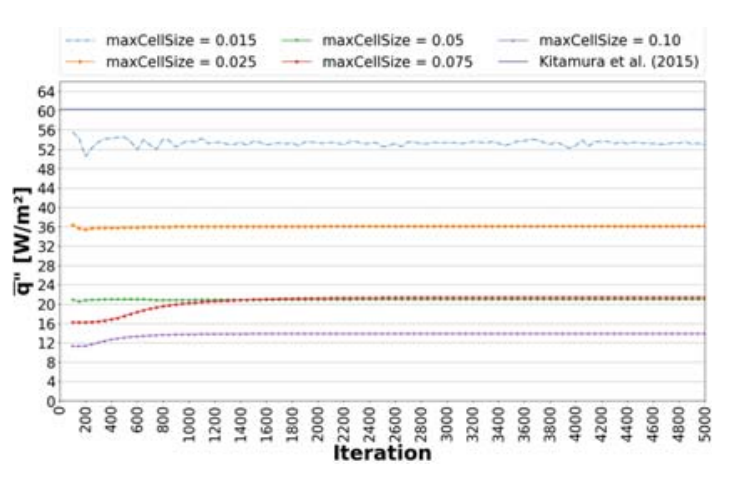

Figure 5. Average convective heat flux over the plate versus iterations number for the different refinement degrees of Mesh B.

According to Kitamura et al. (2015) and from equation 12 , there is the experimental value of $\overline{\mathrm{q}^{\prime \prime}}=$ $60.29 \mathrm{~W} / \mathrm{m}^{2}$ for $\mathrm{Ra}_{\mathrm{d}_{\mathrm{e}}}=3 \cdot 10^{8}$.

The analysis of Fig. 4 shows that the meshes with maxCellSize between 2.00 and 10.00 reach convergence (oscillating type) after approximately 2600 iterations, underestimating $\overline{q^{\prime \prime}}$ in relation to the experimental results. For meshes with maxCellSize between 0.80 and 1.00 a greater number of iterations is necessary to obtain the convergence (oscillating type too), with overestimating $\overline{\mathrm{q}^{\prime \prime}}$. The mesh with maxCellSize equal 0.90 of configuration A shows itself as the best option in accuracy for the analyzed problem situation.

Fig.5 shows that none of the meshes of configuration $\mathrm{B}$, in comparison to the results of configuration A, presented good accuracy to the experimental results of Kitamura et al. (2015). The mesh with maxCellSize equal to 0.015, with the highest refinement level, achieved greater accuracy; but still far from the experimental reference and with an absurdly higher computational cost than any mesh of configuration $\mathrm{A}$. The meshes with maxCellSize equals 0.25 and 0.20 did not show solution convergence, and their results were not shown in Figure 5.

The solution oscillation characteristics of the configuration B meshes, due to the larger amplitude of the scale, proved to be difficult to visualize. However, for the mesh with maxCellSize equal to 0.015 , such behavior is quite pronounced.

Therefore, the mesh configuration B proves to be unfeasible for the studied application.

For the analysis of the $\overline{\mathrm{Nu}}_{\mathrm{d}_{\mathrm{e}}}$ of the different simulated meshes, there are Figures 6 and 7, respectively, for Mesh A and B configurations.

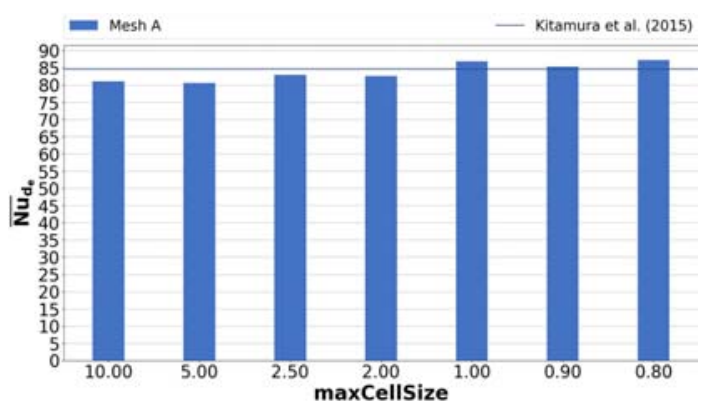

Figure 6. Average Nusselt number versus maxCellSize for the different refinement degrees of Mesh A.

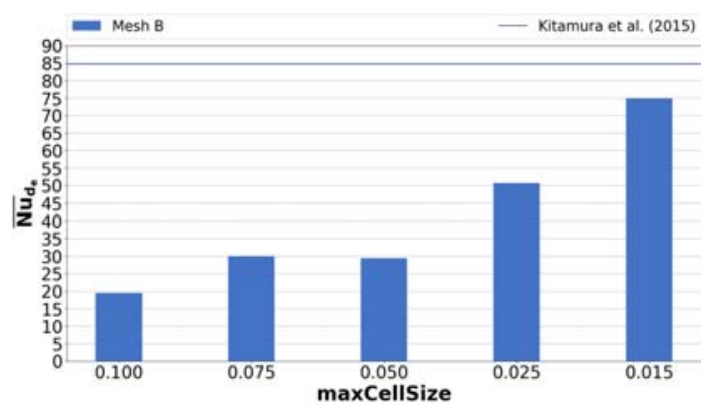

Figure 7. Average Nusselt number versus maxCellSize for the different refinement degrees of Mesh B.

Fig. 6 shows a tendency for stabilization of the values of $\overline{\mathrm{Nu}}_{\mathrm{d}_{\mathrm{e}}}$ around the experimental referential presented, especially under conditions of greater refinement. This demonstrates the good physical representation of mesh A and the boundary conditions applied in representing the studied physical domain. It also demonstrates the characteristics of independence and consistency for the studied meshes.

Fig. 7 confirms the low accuracy behavior already shown in Fig. 5. In addition, there is an increase in precision with an increase in the degree of refinement of the mesh for configuration $\mathrm{B}$. It is also observable that the independence and consistency behavior was not obtained for the studied meshes.

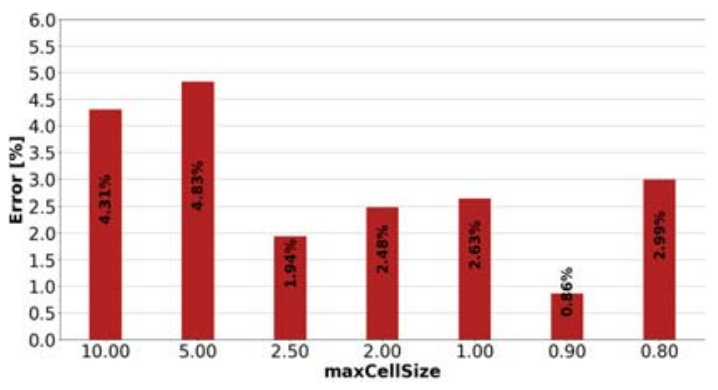

Figure 8. Average relative percentage error versus maxCellSize for the different refinement degrees of Mesh A. 


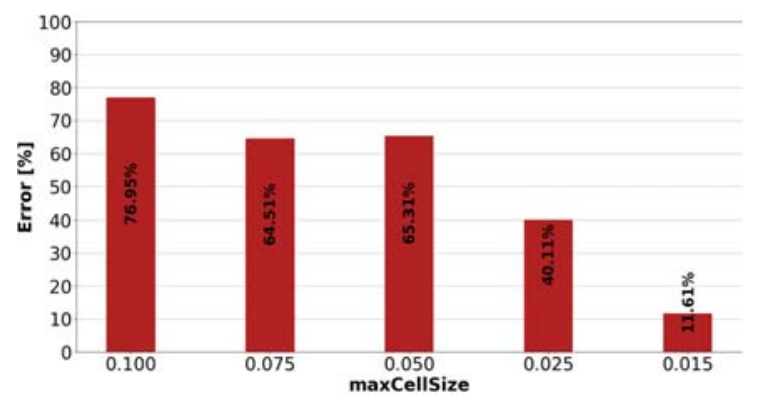

Figure 9. Average relative percentage error versus maxCellSize for the different refinement degrees of Mesh B.

Finally, to analyze the average relative percentage error of the different meshes studied, according to Eq. 13, there are Figures 8 and 9, respectively, for Mesh A and B configurations.

Fig. 8 quantitatively shows the behavior of independence and consistency of the different meshes studied in configuration A. With maximum errors smaller than $5 \%$, any of the mesh configurations is very suitable for the numerical analysis of the studied problem situation. However, in applications requiring greater precision, with maximum errors less than $2.5 \%$, the mesh with maxCellSize equal to 2.50 , in terms of cost-benefit, proves to be the best option.

The use of fine uniform refinement in the plate region in configuration A contributes significantly to reducing errors and using meshes with lower computational costs. This is due to greater gradients of fluid-thermal properties in this region, requiring meshes with greater refinement to correctly capture these properties.

The results discussed for the mesh configuration A experimentally validate, and with excellent accuracy, the boundary conditions and the numerical methodology developed and applied to the problem situation studied.

It is important to highlight that the analysis performed was based on the analysis of heat transfer by natural convection over the plate, through the physical parameters $\overline{\mathrm{q}^{\prime \prime}}$ and $\overline{\mathrm{Nu}}_{\mathrm{d}_{\mathrm{e}}}$. The study of other physical parameters and the turbulent flow regime itself would better support and validate the analysis and selection of the meshes; these are shown as an opportunity for future studies.

Fig. 9 shows and confirms the non-suitability of mesh configuration $\mathrm{B}$, despite being simpler, for the numerical study of the studied problem situation. Only the mesh with maxCellSize equal to 0.015 proved to be a little more adequate; however, compared to the mesh with maxCellSize equal to 2.5 of configuration A, it has an additional 4,617,539 elements and lower relative accuracy of $9.67 \%$, which makes its use completely unfeasible.

The behavior of the curve in Figure 9 shows the error reduction with the increase of the mesh refinement degree. Thus, to obtain solutions with greater accuracy, even more refined computational meshes would be needed (with well over 5 million elements); which is not reasonable and numerically applicable for the studied problem situation.

\section{CONCLUSIONS}

The present study evaluated the influence of two different mesh configurations, at different refinement levels, in predicting turbulent natural convection heat transfer in isothermal flat plates. The boundary conditions used and the methodology of analysis and numerical modeling applied to the problem studied were validated with excellent precision from experimental results by Kitamura et al. (2015).

Mesh A, with non-uniform decreasing staggered and fine uniform refinement in the flat region, proved to be more suitable for accuracy and cost-benefit for the problem situation studied. The consistency and independence characteristics of the studied meshes were confirmed, with an accuracy of $0.86 \%$ for the mesh with maxCellSize equal to 0.90 and 2,044,144 elements.

The Mesh Configuration B, totally uniform, did not prove suitable for the problem situation studied. The high dependence of accuracy on the degree of refinement of the mesh and the extremely high computational cost of the simulations, made it unfeasible to use this type of mesh; despite its greater topological simplicity.

Finally, the use of totally free and open-source numerical tools in developing research in the CFD area is shown as a trend and an alternative to current budget constraints in the education, science, and technology sectors.

\section{ACKNOWLEDGEMENTS}

The author acknowledges with gratitude the financial support granted by Instituto Federal de Educação, Ciência e Tecnologia São Paulo (IFSP), Araraquara campus.

\section{REFERENCES}

Bergman, T. L., Lavine, A. S., Incropera, F. P. and De Witt, D. P., 2014, Fundamentos da Transferência de Calor e de Massa, LTC (in Portuguese).

Bird, R. B., Stewart, W.E. and Lightfoot, E. N., 2004, Transport Phenomena, John Wiley \& Sons.

Boz, Z., Erdogdu, F. and Tutar, M., 2014, Effects of Mesh Refinement, Time Step Size and Numerical Scheme on The Computational Modeling of Temperature Evolution During NaturalConvection Heating, Journal of Food Engineering, Vol. 123, pp. 8-16.

Frank, A., Heidemann, W. and Spindler, K. 2019, Electronic Component Cooling Inside Switch Cabinets: Combined Radiation and Natural 
Convection Heat Transfer, Heat Mass Transfer, Vol. 55, pp. 699-709.

Juretic, F., 2015, User Guide cfMesh v1.1. Creative Fields, Zagreb, Croatia. URL: http://cfmesh.com/wp-content/uploads/2015/09/User Guide-cfMesh_v1.1.pdf. Retrieved 15 June 2021. Jayatilleke, C. L. V., 1966, The Influence of Prandtl Number and Surface Roughness on The Resistance of The Laminar Sublayer to Momentum and Heat Transfer, Ph.D. Thesis, Imperial College of Science and Technology, London.

Kitamura, K., Mitsuishi, A., Suzuki, T. and Kimura, F., 2015, Fluid Flow and Heat Transfer of Natural Convection Adjacent to Upward-Facing, Rectangular Plates of Arbitrary Aspect Ratios, International Journal of Heat and Mass Transfer, Vol. 89, pp. 320-332.

Machado, H. A. and Ramos, A. G., 2006, Performance Analysis of Thermal Diodes, Revista de Engenharia Térmica, Vol. 5, No. 2, pp. 66-71.

Mariani, V. C. and Belo, I. M., 2006, Numerical Studies of Natural Convection in a Square Cavity, Revista de Engenharia Térmica, Vol. 5, No. 1, pp. 6872.

Menter, F., Kuntz, M. and Langtry, R., 2003, Ten Years of Industrial Experience with the SST Turbulence Model, in: International Symposium on Turbulence: Heat and Mass Transfer, Begell House, Antalya, pp. 625-632.

Moukalled, F., Mangani, L. and Darwish, M., 2015, The Finite Volume Method in Computational Fluid Dynamics: An Advanced Introduction with OpenFOAM ${ }^{\circledR}$ and Matlab ${ }^{\circledR}$, Springer.

Norton, T. and Sun, D.W., 2006, Computational Fluid Dynamics (CFD) - An Effective and Efficient Design and Analysis Tool for the Food Industry: A Review, Trends in Food Science \& Technology, Vol. 17, No. 11, pp. 600-620.

OpenCFD, 2020, OpenFOAM: The Open Source CFD Toolbox, User Guide (v2012), OpenCFD Ltd.

Padilla, E. L., M. Campregher, R. and Silveiraneto, A., 2006, Numerical Analysis of the Natural Convection in Horizontal Annuli at Low and Moderate Ra, Revista de Engenharia Térmica, Vol. 5, No. 2, pp. 58-65.

Pope, S. B., 2000, Turbulent Flows, England: Cambridge University Press,

Silva, V. A., Anselmo, B. C. S., Silva, A. L. F. L., Silva and S. M. M. L., 2016, Experimental Analysis of the Influence of Heat Sink Geometric Parameters on Natural Convection, Revista de Engenharia Térmica, Vol. 15, No. 1, pp. 26-32.

Tu, J., Yeoh, G.H. and Liu, C. 2008, Computational Fluid Dynamics: A Practical Approach, Elsevier Applied Science Publishers.

Verdério Júnior, S. A., Scalon, v. L. and Padilha, A., 2015, Influence of Modeling of Turbulence in the Flow Parameters Within a Food Oven Using the
OPENFOAM ${ }^{\circledR}$, Revista de Engenharia Térmica, Vol. 14, No. 2, pp. 69-76.

Versteeg, H. K. and Malalasekera, W., 2007, An Introduction to Computational Fluid Dynamics - The Finite Volume Method, Longman Scientific and Technical. 